\title{
Markovian Behaviour and Constrained Maximization of the Entropy in Chaotic Quantum Systems
}

\author{
A. Romanelli ${ }^{\text {a }}$ A.C. Sicardi Schifino ${ }^{a, 1}$ G. Abal ${ }^{\text {a }}$ R. Siri ${ }^{\text {a }}$ \\ R. Donangelo ${ }^{b}$ \\ ${ }^{a}$ Instituto de Física, Facultad de Ingeniería \\ Universidad de la República \\ C.C. 30, C.P. 11000, Montevideo, Uruguay \\ ${ }^{\mathrm{b}}$ Instituto de Física, Universidade Federal do Rio de Janeiro \\ C.P. 68528, 21945-970 Rio de Janeiro,Brazil
}

\begin{abstract}
The separation of the Schrödinger equation into a Markovian and an interference term provides a new insight in the quantum dynamics of classically chaotic systems. The competition between these two terms determines the localized or diffusive character of the dynamics. In the case of the Kicked Rotor, we show how the constrained maximization of the entropy implies exponential localization.
\end{abstract}

Key words: kicked rotor; markovian process; dynamical localization

In the last few decades the field of Quantum Chaos has drawn the attention of researchers in several areas of science. Many interesting phenomena were studied in systems such as atom traps and microwave cavities. Furthermore, the recent advances in technology that allow to construct and almost perfectly preserve quantum states, has opened the field of quantum computation, where chaotic effects and their control play an essential role (1).

In this work we develop a different and general approach to the subject of dynamical localization (DL) and the related issue of quantum diffusion. This approach leads to an improved understanding of why DL takes place in some systems while in others quantum diffusion continues for ever. The path we follow consists in rewriting the Schrödinger equation in a form in which the part responsible for DL is separated from the part responsible for quantum

$\overline{1}$ also at: Facultad de Ciencias, Universidad de la República. 
diffusion. As we shall see, the terms responsible for quantum diffusion have the form of a master equation, typical of Markovian processes, while the other part consists of interference terms required to preserve the unitary character of the quantum evolution. The master equation has been extensively used by W. Zurek and co-workers (2) to study the emergence of a classical behavior due to environment-induced decoherence. For the systems considered in the present work the environment does not play a role, and the decoherence, if present, is self-generated by the dynamics.

Consider a quantum system described by a generic time-dependent Hamiltonian of the form $H(t)=H_{0}+V(t)$, where $H_{0}$ is the time-independent part, with known eigenstates and eigenvalues satisfying $H_{0}|k\rangle=E_{k}|k\rangle$. We write the wave function as $|\Psi(t)\rangle=\sum_{k} a_{k}(t)|k\rangle$. The time-dependent part of the Hamiltonian, $V(t)$, induces transitions between the eigenstates of $H_{0}$. The actual quantum unitary evolution of $|\Psi\rangle$ is given by the Schrödinger equation. However, if discrete times $t_{n}$ are considered, then a quantum map is obtained

$$
a_{k}\left(t_{n+1}\right)=\sum_{l} U_{k l}^{(n)} a_{l}\left(t_{n}\right)
$$

where $U_{k l}^{(n)} \equiv\left\langle k\left|U\left(t_{n+1}, t_{n}\right)\right| l\right\rangle$ and $U\left(t_{n+1}, t_{n}\right)$ is the evolution operator connecting the state at time $t_{n}$ with the one at time $t_{n+1}$. In terms of $U$, the occupation probability $P_{k}\left(t_{n}\right) \equiv\left|a_{k}\left(t_{n}\right)\right|^{2}$ can be expressed as

$$
P_{k}\left(t_{n+1}\right)=\sum_{l, m} U_{k l}^{(n)} U_{k m}^{(n) *} a_{l}\left(t_{n}\right) a_{m}^{*}\left(t_{n}\right)
$$

If we separate the diagonal terms in its rhs, eq. (2) may be written as

$$
P_{k}\left(t_{n+1}\right)=\sum_{l} T_{k l} P_{l}\left(t_{n}\right)+\beta_{k}\left(t_{n}\right)
$$

with

$$
\beta_{k}\left(t_{n}\right)=\sum_{\substack{l, m \\(l \neq m)}} U_{k l}^{(n)} U_{k m}^{(n) *} a_{l}\left(t_{n}\right) a_{m}^{*}\left(t_{n}\right),
$$

where we have defined $T_{k l} \equiv\left|U_{k l}^{(n)}\right|^{2}$ as the transition probability. This is meaningful because $T_{k l} \geq 0$ and $\sum_{k} T_{k l}=\sum_{l} T_{k l}=1$. Thus, if the term $\beta_{k}$ could be neglected in eq. (3), the time evolution of the occupation probability would be described by a Markovian process in which the transition probability $k \rightarrow l$, in a time $\Delta t_{n}=t_{n+1}-t_{n}$, is given by $T_{k l}$. 
We now consider the transition probability per unit time, defined as

$$
W_{k l} \equiv \frac{T_{k l}-\delta_{k l}}{\Delta t_{n}}
$$

where $\delta_{k l}$ is the Kronecker delta. A straightforward calculation shows that the quantum evolution equation (3), can be written as

$$
P_{k}\left(t_{n+1}\right)=P_{k}\left(t_{n}\right)+\sum_{l \neq k}\left(W_{k l} P_{l}-W_{l k} P_{k}\right) \Delta t_{n}+\beta_{k}
$$

In this form, two qualitatively different terms can be distinguished: one associated with a Markovian process, i.e. a classical-like diffusion, and the other, $\beta_{k}$, associated to quantum interference effects. This last term preserves the unitary character of the evolution. Its contribution depends on the continuous or discrete character of the dynamical response spectrum $(3 ;$; $;$ 5). For systems with a discrete spectrum, as the periodic kicked rotor, it is of the same order of magnitude as the Markovian term, and becomes responsible for the dynamical localization found in such systems. A finite time of the order of $1 / \Delta \omega$, where $\Delta \omega$ is the average separation in the frequency response spectrum, is required in order to resolve the discreteness of the spectrum. At shorter time scales, the discreteness of the spectrum has no effect on the dynamics, the Markovian approximation holds and the system "mimics" classical chaos. In the case of a continuous spectrum, the terms contributing to $\beta_{k}$ in eq. (4) are scattered on the complex plane in such a way that their sum becomes negligible compared to the Markovian term in eq. (6). Then, the unitary evolution is well approximated, for arbitrary long times, by a Master equation

$$
\frac{\partial P_{k}}{\partial t_{n}}=\sum_{l \neq k}\left(W_{k l} P_{l}-W_{l k} P_{k}\right)
$$

which results from eq. (6) if $\beta_{k}$ is neglected.

Assuming, for simplicity, that $W_{k, k-l}=W_{k, k+l}$, eq. (7) may be written as a diffusion equation,

$$
\frac{\partial P_{k}}{\partial t_{n}}=\frac{D}{2} \frac{\partial^{2} P_{k}}{\partial k^{2}}
$$

where the diffusion coefficient is

$$
D=2 \sum_{l=1}^{\infty} W_{k, k+l} l^{2} .
$$

The differential operators in eqs. (7) and (8) represent the discrete derivatives $\partial P_{k} / \partial t_{n}=\left[P_{k}\left(t_{n+1}\right)-P_{k}\left(t_{n}\right)\right] / \Delta t_{n}$ and $\partial^{2} P_{k} / \partial k^{2}=\left[P_{k+l}\left(t_{n}\right)+P_{k-l}\left(t_{n}\right)-2 P_{k}\left(t_{n}\right)\right] / l^{2}$. Note that, according to eq. (8), an initial gaussian distribution evolves as a spreading gaussian. 
As it is well known (6), a Markovian process described by a Master equation such as (7), satisfies the H-theorem, which assures that the thermodynamic entropy, $S \equiv-\sum_{k} P_{k} \ln P_{k}$ can only increase. On the other hand, in a unitary evolution the entropy calculated from the density operator, $\mathbf{S} \equiv-\operatorname{trace}(\rho \ln \rho)$, must be constant. We note that this expression is equivalent to the entropy $S$ only if the index $k$ can be associated to the eigenstates of the complete Hamiltonian $H(t)$. Obviously in this situation the Master equation does not hold. Since in our case we have used the representation of eigenstates of $H_{0}$, and not of $H(t)$, the thermodynamical entropy $S$ corresponds to a "coarse-graining" of the entropy $\mathbf{S}$. In the rest of this paper, we refer to the thermodynamic entropy $S$ simply as "entropy". Even though it is possible to define quantum dynamical entropies (7), as long as the Master equation (7) holds it is adequate to use the associated thermodynamical entropy $S$.

The introduction of the entropy $S$ will allow us to use thermodynamic arguments to draw interesting conclusions regarding DL. We recall (8) that if the value of an observable $M$ in state $|k\rangle$ is $M_{k}$, the maximum value of the entropy consistent with a given constraint,

$$
\sum_{k} M_{k} P_{k}=\langle M\rangle
$$

corresponds to an exponential (canonical) distribution

$$
P_{k}=e^{-\Omega} e^{-\lambda M_{k}} .
$$

This equilibrium distribution is attained after a diffusive process in which the entropy is maximized. The Lagrange multipliers $\lambda, \Omega$ are determined by the constraint (10) and the normalization condition $\sum_{k} P_{k}=1$, respectively. In particular, the condition $\frac{\partial \Omega}{\partial \lambda}+\langle M\rangle=0$ must be satisfied. However, if the constraint (10) is removed, the distribution evolves according to a diffusion equation for arbitrary long times and the entropy increases without bound, provided that the spectrum of $H_{0}$ is unbounded.

At this point, it is convenient to consider a concrete example. We choose the quantum Kicked Rotor (QKR), one of the first classically chaotic systems to be quantum-mechanically investigated (9). For this system, the evolution operator has the following form (10)

$$
U_{k l}^{(n)}=i^{-(l-k)} J_{l-k}(\kappa) e^{-i E_{l} \Delta t_{n} / \hbar},
$$

where $\kappa=K / \hbar$ is the dimensionless kick strength, $E_{l}=\hbar^{2} l^{2} / 2 I$ is the kinetic energy of the rotor, $I$ its moment of inertia, $\Delta t_{n}=t_{n+1}-t_{n}=T$ the constant time interval between kicks and $J_{s}$ is the $s^{\text {th }}$ order Bessel function. Note that in this case, the condition $W_{k, k-l}=W_{k, k+l}$ is satisfied by (12). Then, as long as the term $\beta_{k}$ can be neglected in eq. (6), the evolution is given by the diffusion 
equation (8). The diffusion coefficient, obtained from eq. (9) using standard properties of the Bessel functions, is $D=\kappa^{2} / 2 T$, which is consistent with the classical result.

The average energy of the QKR is given by $E\left(t_{n}\right)=\sum_{k=-\infty}^{\infty} E_{k} P_{k}\left(t_{n}\right)$. A direct calculation based on eq. (6), shows that the energy change across two consecutive kicks, $\Delta E_{n} \equiv E\left(t_{n+1}\right)-E\left(t_{n}\right)$, can be written in terms of the transition probabilities $W_{k l} \Delta t_{n}$ as

$$
\begin{aligned}
\Delta E_{n} & =\sum_{k, l=-\infty}^{\infty} E_{l} P_{k}\left(t_{n}\right) W_{k l} \Delta t_{n}+\sum_{l=-\infty}^{\infty} E_{l} \beta_{l} \\
& =\frac{K^{2}}{4 I}+\sum_{l=-\infty}^{\infty} E_{l} \beta_{l}\left(t_{n}\right) .
\end{aligned}
$$

The first term in the rhs of this expression describes the classical diffusive increase in the energy while the second term, involving the sum of $\beta_{l}$, accounts for quantum interference effects and is calculated explicitly in (5). In Fig. 1, we show the contribution of each term in eq. (13) to the energy of the QKR, as a function of the number of kicks, $n$. The dotted line in this figure corresponds to the contribution of the interference term, $\sum_{l} E_{l} \beta_{l}\left(t_{n}\right)$ and the dashed line to the contribution of the Markovian term. This last contribution has a slope $\kappa^{2} / 2$ and is coincident with the classical linear diffusive increase. After $\sim 50$ kicks, the cumulative effect of the quantum coherence begins to cancel out the diffusive growth of the energy. In eq. (13), $\Delta t_{n}=T$ and the parameters are $\kappa=21.0$ and $\hbar T / I=1$. The energy is in units of $\hbar^{2} / 2 I$ and the smoothing was obtained by averaging over one hundred initial conditions, corresponding to the fifty lowest eigenvalues of $H_{0}$. We see that the contribution of the interference term is negligible until dynamical localization sets in, so that this term may be identified as the one responsible for DL.

During the quantum diffusion which precedes DL, when the interference term is negligible, the quantum evolution mimics a Markovian process. and the entropy increases, as shown in Fig. 2. However, the discrete quasi-energy spectrum of the QKR Floquet operator (12) introduces a constraint that limits the entropy increase. In fact, as is well known (10), only a finite number $\left(L_{0}\right.$ on the average) of eigenstates of $H_{0}$ are required to describe an eigenstate of the Floquet operator. If we initialize the system in the eigenstate $\left|k_{0}\right\rangle$ of $H_{0}$, the subsequent evolution can only involve eigenstates $|k\rangle$ of $H_{0}$ such that $\left|k-k_{0}\right| \leq L$. This fact represents a constraint on the entropy increase, of the type given by eq. (10). The actual value of $L$ depends on the initial condition, but it must obey $\langle L\rangle=L_{0}$, where the average is over different initial states $\left|k_{0}\right\rangle$. For each $k_{0}$, we take $L$, i.e. the maximum distance $\left|k-k_{0}\right|$ in angular momentum space present in the dynamical evolution, as the observable $M$ of eq. (10). Then, the corresponding constraint is $\langle L\rangle=\left\langle\left|k-k_{0}\right|_{\text {max }}\right\rangle=L_{0}$. From this constraint 


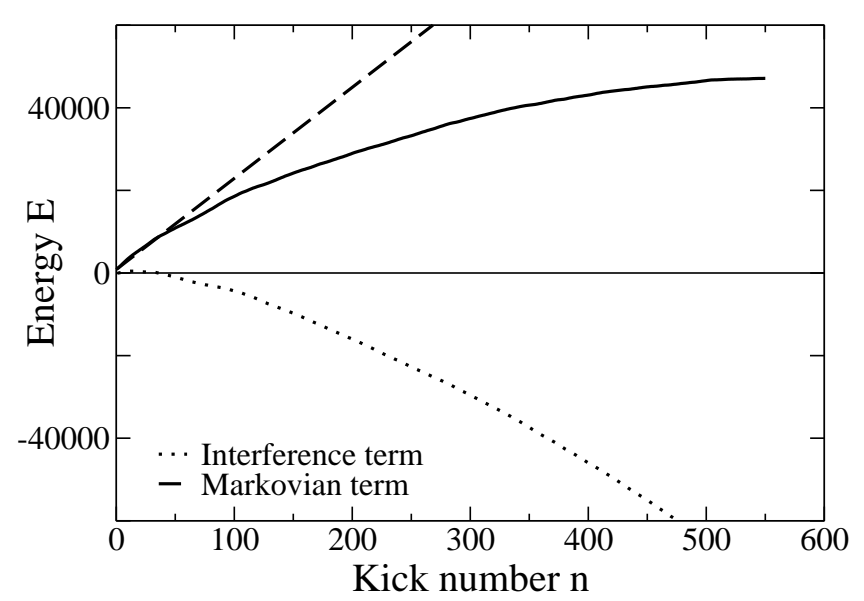

Fig. 1. Contribution of each of the terms of eq. (13) to the average energy of the kicked rotor. See text for details.

and the normalization condition for the occupation probability, we obtain the Lagrange multipliers $\lambda=\sinh ^{-1}\left(1 / L_{0}\right), \Omega=\ln \left[L_{0}+\left(L_{0}^{2}+1\right)^{1 / 2}\right]$. Then, the probability distribution (11) resulting from the constrained maximization of the entropy is

$$
P_{k}=\frac{e^{-\lambda\left|k-k_{0}\right|}}{L_{0}+\left(L_{0}^{2}+1\right)^{1 / 2}} \approx \frac{1}{2 L_{0}} e^{-\frac{\left|k-k_{0}\right|}{L_{0}}}
$$

because $L_{0} \gg 1$. This exponential profile for the localized wave function has been verified both numerically (10) and experimentally (11) for the case of the QKR. When the maximum value of the entropy consistent with this constrain is attained, the interference term in the rhs of eq. (6) becomes non-negligible and so the master equation approximation does not hold. Then the entropy remains constant (see Fig. 2).

The origin of DL may thus be traced to the discrete nature of the dynamical response frequency spectrum (or equivalently for the QKR, the quasi-energy spectrum), which determines the degree of randomness of the $\beta_{k}$ values in the complex plane. This is discussed in further detail in (5).

To clarify the relation of DL to the behavior of the second term in the rhs of eq. (13) we now consider a simple modification of the QKR, in which the time intervals $\Delta t_{n}$, are randomly chosen. In this case eqs. (12) and (13) are valid, but the contribution of the interference term in the rhs of eq. (6) is negligible for arbitrarily long times since the dynamical response frequency spectrum is continuous and this system does not show DL (5). In this case, the evolution is always Markovian and it can be described by the diffusion equation (8) with the diffusion coefficient given by eq. (9). 


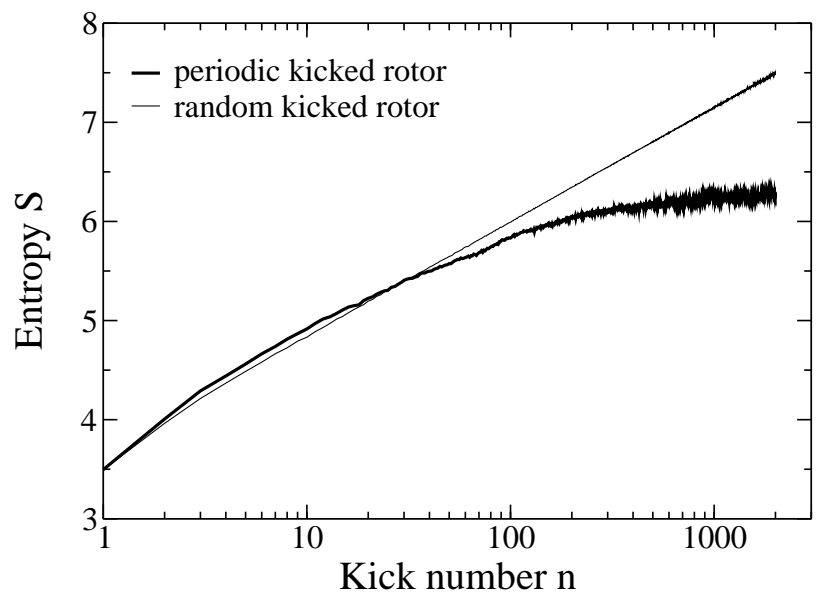

Fig. 2. Entropy $S=-\sum_{k} P_{k} \ln P_{k}$ as a function of the kick number (log scale) for the quantum kicked rotor with a fixed time interval between kicks (thick line) and a random time interval between kicks (thin line). The parameters and averaging over initial conditions are the same as in Fig. 1.

One might think that these diffusive dynamics is an artifact resulting from the randomness of the time intervals between successive kicks. That this is not the case may be seen by considering the double Kicked Rotor (QDKR), a quasiperiodic version of the QKR. The QDKR is obtained when a second series of pulses is applied to the rotor, with an irrational ratio between the periods of both trains of pulses. The evolution operator (12) also holds for the QDKR, but the time intervals $\Delta t_{n}$ now form a pseudo-random sequence. The response spectrum of this system is dense and it undergoes unlimited quantum diffusion at a rate consistent with the classical diffusion rate (5). This implies that the contribution of the interference term in eq. (6) is negligible also in this case. In both cases, the probability distribution $P_{k}\left(t_{n}\right)$ evolves as a spreading gaussian. The long-term evolution of the entropy is markedly different for these systems than for the periodic case. In Fig. 2, the time evolution of the entropy $S$ for the QKR and the random kicked rotor are compared. In the case of the QKR, the entropy stops growing when the constraint is satisfied, while it grows without bound in the other case.

Finally, we remark that the approach of separating the Schrödinger equation into a master equation part supplemented by a term which takes into account quantum interference effects, provides a new insight on quantum diffusion and dynamical localization. As we have shown, the localized or diffusive character of the dynamics can be understood as the result of the competition between these terms of the evolution equation. The Markovian term dominates the dynamics for a time which depends on the topology of the dynamical response spectrum. The general procedure has been illustrated using the quantum kicked rotor, in its periodic, quasi-periodic and random kick ver- 
sions. In particular, we have explained the exponentially localized probability distribution of the QKR using simple, well accepted results from the theory of stochastic processes. This exponential distribution results from the constrained maximization of the coarse-grained entropy. The localization-length constraint is due to the discrete nature of the dynamical response spectrum of the QKR. In the other cases considered the dynamical response spectrum is dense, the localization length is infinite (i.e. the constraint is absent) and the entropy increase continues indefinitely, as is characteristic of a diffusive process. We have shown, that in this case the Schrödinger equation is equivalent to a diffusion equation with the classical diffusion coefficient.

The approach that we have presented here may be used to describe the dynamics of an arbitrary quantum system in which there is quantum diffusion.

We acknowledge the support of PEDECIBA and CONICYT-Clemente Estable (project \#6026), RD acknowledges partial financial support from $M C T / F I N E P / C N P q(P R O N E X)$ under contract 41.96.0886.00.

\section{References}

[1] Quantum Computation and Quantum Information, M.A. Nielsen and I.L. Chuang, Cambridge University Press, Cambridge (2000).

[2] S. Habib, K. Shizume and W.H. Zurek, Phys. Rev. Lett. 804361 (1998); W.H. Zurek and J.P. Paz, Phys. Rev. Lett. 722508 (1994); J.P. Paz, S. Habib and W.H. Zurek, Phys. Rev. D 47488 (1993); W. Zurek, Phys. Today, 36, October 1991.

[3] S. R. Jain, Phys. Rev. Lett. 703553 (1993); Nucl. Phys. A673 423 (2000).

[4] M. Feingold and A. Peres, Phys. Rev. A 34591 (1986).

[5] G. Abal, R. Donangelo, A. Romanelli, A.C. Sicardi Schifino and R. Siri, Phys. Rev. E 65, 046236 (2002).

[6] N.G. van Kampen Stochastic Processes in Physics and Chemistry, NorthHolland (1981).

[7] R. Alicki and M. Fannes, Lett. Math. Phys. 3275 (1994).

[8] H. Haken, Synergetics-An Introduction, Springer-Verlag (1983).

[9] G. Casati, B.V. Chirikov, F.M. Izrailev and J. Ford, Lect. Notes Phys. 93334 (1979).

[10] F.M. Izrailev, Phys. Rep. 196, 299 (1990).

[11] F.L. Moore, J.C. Robinson, C. Bharucha, B. Sundaram and M.G. Raizen, Phys. Rev. Lett. 75, 4598 (1995).; F.L. Moore, J.C. Robinson, C.F. Bharucha, P.E. Williams and M.G. Raizen, Phys. Rev. Lett. 73, 2974 (1994). 